\title{
PROCESSO DE ENFERMAGEM: DA TEORIA À PRÁTICA ASSISTENCIAL E DE PESQUISA
}

\author{
Nursing Process: from theory to the practice of care and research \\ Proceso de Enfermería: de la teoría a la práctica asistencial y de \\ investigación
}

Telma Ribeiro Garcia ${ }^{1}$

Maria Miriam Lima da Nóbrega ${ }^{2}$

\begin{abstract}
RESUMO
Define-se o Processo de Enfermagem como um instrumental tecnológico ou um modelo metodológico para o cuidado profissional de enfermagem. Descreve-se a evolução do conceito e como o Processo de Enfermagem avançou, da ênfase inicial na identificação e resolução de problemas para o esforço de identificação e classificação de diagnósticos de enfermagem e, mais atualmente, para a especificação e verificação, na prática, de resultados do paciente que sejam sensíveis às intervenções de enfermagem. Por fim, apresentam-se exemplos de estudos que vinculam os elementos da prática profissional, inerentes ao Processo de Enfermagem (diagnósticos, intervenções e resultados de enfermagem), à investigação científica.
\end{abstract}

Palavras-chave: Enfermagem. Processos de Enfermagem. Prática Profissional.

\begin{abstract}
The Nursing Process is defined as a technological instrument or a methodological model for nursing professional care. The evolution of the concept is described, as the Nursing Process moved forward, from the initial emphasis in the identification and resolution of problems, to the effort of identification and classification of nursing diagnoses and, more currently, to the specification and clinical testing of the patient's results that are sensible to the nursing interventions. Finally, examples of studies that link the elements of the professional practice, inherent to the Nursing Process (nursing diagnoses, interventions and outcomes), to the scientific research presented.
\end{abstract}

Keywords: Nursing. Nursing Process. Professional practice.

\section{Resumen}

Se define el Proceso de Enfermería como un instrumento tecnológico o un modelo metodológico para el cuidado profesional de enfermería. Se describe la evolución del concepto, y como el Proceso de Enfermería avanzó, del énfasis inicial en la identificación y resolución de problemas, al esfuerzo de identificación y clasificación de los diagnósticos de enfermería y, más actualmente, a la especificación y la comprobación clínica de los resultados del paciente que son sensibles a las intervenciones de enfermería. Finalmente, se presentan ejemplos de estudios que vinculan los elementos de la práctica profesional, inherentes al Proceso de Enfermería (diagnósticos, intervenciones y resultados de enfermería), a la investigación científica.

\footnotetext{
'Professora Adjunta IV (aposentada) do Departamento de Enfermagem de Saúde Publica e Psiquiatria. Docente do Programa de Pós-Graduação em Enfermagem - Centro de Ciências da Saúde, UFPB. Pesquisadora do CNPq. Brasil. E-mail: telmagarciapb@gmail.com, ${ }^{2}$ Professora Associada do Departamento de Enfermagem de Saúde Pública e Psiquiatria. Docente do Programa de Pós-Graduação em Enfermagem - Centro de Ciências da Saúde, UFPB. Pesquisadora do CNPq. Brasil E-mail: miriam@ccs.ufpb.br
} 


\section{INTRODUCÃO}

0 Processo de Enfermagem indica um trabalho profissional específico e pressupõe uma série de ações dinâmicas e interrelacionadas para sua realização, ou seja, indica a adoção de um determinado método ou modo de fazer (Sistematização da Assistência de Enfermagem), fundamentado em um sistema de valores e crenças morais e no conhecimento técnico-científico da área. Na literatura, costuma ser descrito como o ponto focal, o cerne ou a essência da prática da Enfermagem. Entretanto, a compreensão acerca do significado e sua adoção deliberada na prática profissional ainda não são unanimidade no âmbito da Enfermagem, embora se observe ter havido uma sensível mudança nesse sentido a partir das três últimas décadas do século XX1.

Compreendendo-se tecnologia como a utilização do conhecimento para a produção de bens e serviços ${ }^{2}$, pode-se definir o Processo de Enfermagem como: 1) um instrumento tecnológico de que lançamos mão para favorecer o cuidado, para organizar as condições necessárias à realização do cuidado e para documentar a prática profissional; ou 2) um modelo metodológico que nos possibilita identificar, compreender, descrever, explicar e/ou predizer as necessidades humanas de indivíduos, famílias e coletividades, em face de eventos do ciclo vital ou de problemas de saúde, reais ou potenciais, e determinar que aspectos dessas necessidades exigem uma intervenção profissional de enfermagem ${ }^{1,3}$.

A implementação do Processo de Enfermagem demanda habilidades e capacidades cognitivas, psicomotoras e afetivas, que ajudam a determinar o fenômeno observado e o seu significado; os julgamentos que são feitos e os critérios para sua realização; e as ações principais e alternativas que 0 fenômeno demanda, para que se alcance um determinado resultado. Esses aspectos dizem respeito aos elementos da prática profissional considerados, por natureza, inseparavelmente ligados ao Processo de Enfermagem: o que os agentes da Enfermagem fazem (ações e intervenções de enfermagem), tendo como base o julgamento sobre necessidades humanas específicas (diagnóstico de enfermagem), para alcançar resultados pelos quais se é legalmente responsável (resultados de enfermagem) ${ }^{4}$.

Os elementos inerentes à prática profissional (diagnósticos, intervenções e resultados de enfermagem) favoreceram o desenvolvimento, em curso, de sistemas de classificação de conceitos que fazem parte da linguagem profissional da área, instrumentos tecnológicos a serem utilizados: a) no processo e no produto do raciocínio e julgamento clínico acerca das necessidades humanas de indivíduos, famílias e coletividades, diante de eventos do ciclo vital ou de problemas de saúde, reais ou potenciais; b) no processo e no produto do raciocínio e julgamento terapêutico acerca das necessidades de cuidado da clientela (indivíduos, famílias e coletividades) e dos resultados que são sensíveis à intervenção de enfermagem; e c) na documentação da prática profissional ${ }^{1,3}$.
Tendo por base os aspectos apontados, objetivamos neste artigo fazer uma síntese da evolução do conceito e apresentar exemplos de estudos em que se vinculam os elementos da prática profissional, inerentes ao Processo de Enfermagem (diagnósticos, intervenções e resultados de enfermagem), à investigação científica.

\section{Evolução do conceito Processo de Enfermagem}

$\mathrm{Na}$ Enfermagem, as raízes plantadas por Florence Nightingale têm permitido, até os dias atuais, que se avance no conhecimento sobre o processo de cuidar, considerado a essência do saber e do fazer de seus agentes ${ }^{5}$.

0 significado atribuído ao Processo de Enfermagem e 0 modo como ele é aplicado à prática profissional são dinâmicos, modificando-se ao longo do tempo e de acordo com os diferentes cenários da prática assistencial. Assim, podem ser identificadas gerações distintas do Processo de Enfermagem, cada uma delas influenciada pelo estágio do conhecimento e pelas forças atuantes que lhe são contemporâneos ${ }^{6}$.

A expressão Processo de Enfermagem ainda não era utilizada na segunda metade do século XIX, muito embora, à época, Florence já enfatizasse a necessidade de ensinar as enfermeiras a observar e a fazer julgamentos sobre as observações feitas ${ }^{7}$. Sua introdução formal na linguagem profissional ocorreu nos anos 50 do século XX, sob influência do método de solução de problemas, cujas raízes eram o método científico de observação, mensuração e análise de dados. Observa-se, nesse momento histórico, a ênfase no ensino do método de solução de problemas nas escolas de enfermagem, durante o que se destacava a importância da coleta sistemática e análise de dados, realizadas com rigor metodológico ${ }^{6}$. São exemplos desse modo de pensar a lista dos 21 problemas que deveriam ser o foco do cuidado de enfermagem, elaborada por Faye Abdellah em 1960, e a lista das 14 áreas de necessidades humanas básicas, descrita por Virgínia Henderson, também em 1960. À época, foram, ainda, publicados na literatura da área exemplos de instrumentos de coleta de dados, como 0 modelo baseado em 13 áreas funcionais, proposto por Faye McCain em 1965.

Em 1967, o Processo de Enfermagem foi descrito por Helen Yura e Mary B. Walsh com quatro fases: coleta de dados, planejamento, intervenção e avaliação. Ao descrevê-lo, as duas autoras enfatizaram as habilidades intelectuais, interpessoais e técnicas que consideravam ser necessárias e essenciais à prática profissional e, portanto, aspectos significativos para a execução do Processo de Enfermagem.

A despeito de sua indiscutível importância, um aspecto que caracterizou essa primeira geração do Processo de Enfermagem foi que as necessidades de cuidado e os processos de solução dos problemas dos pacientes relacionavam-se, predominantemente, a determinadas condições fisiopatológicas, médicas ${ }^{6}$. 
Em 1973, foi realizada a primeira conferência para classificação de diagnósticos de enfermagem em que, usando tanto o processo de raciocínio dedutivo quanto o indutivo, as participantes elaboraram e aprovaram a primeira listagem de problemas/situações que eram reconhecidos na prática como pertencentes ao domínio independente da profissão $0^{1,3}$.

0 termo diagnóstico estava presente na literatura da área desde 1950, quando Louise McManus, em conferência pronunciada no Teachers College, em Nova lorque, se referiu à função específica da enfermeira como sendo a identificação ou diagnóstico do problema e o reconhecimento de seus aspectos inter-relacionados, assim como a decisão sobre as ações a serem implementadas para sua solução. Entretanto, até 1973, a etapa diagnóstica não estava incluída no Processo de Enfermagem. Em vista desse fato, pode-se afirmar que 0 movimento de identificação e classificação de diagnósticos de enfermagem marcou o início de uma nova geração do Processo de Enfermagem e, acima de tudo, o início de uma nova era para a Enfermagem, que avança progressivamente, desde então, para sua estruturação definitiva como uma Ciência ${ }^{1,3}$.

O movimento de identificação e classificação de diagnósticos de enfermagem provocou uma revolução no pensamento da área e uma consequente mudança, da ênfase anterior na identificação e solução de problemas para a ênfase no raciocínio diagnóstico e no pensamento crítico. Esse fato muda 0 entendimento do Processo de Enfermagem, de um processo lógico, linear, de solução de problemas, para um processo dinâmico, em espiral ascendente e recorrente, que nos auxilia a gerenciar a informação sobre a clientela e a tomar decisões sobre as ações e intervenções profissionais que ela demanda ${ }^{6}$.

A segunda geração do Processo de Enfermagem determina novas necessidades no ensino e na prática assistencial, em especial a de entender como o julgamento clínico se processa e aumentar a habilidade profissional no raciocínio diagnóstico. Ao término da década de 1980, a evolução e o desenvolvimento contínuo dos sistemas de classificação dos elementos da prática profissional, as pesquisas em andamento sobre a dinâmica do raciocínio e julgamento clínico, bem como a tendência emergente no sistema de saúde para especificar e avaliar os resultados da atenção à saúde, determinaram as condições para a ocorrência de outra transformação no modo de pensar e no modo de aplicar o Processo de Enfermagem 6 . 0 foco da terceira geração do Processo de Enfermagem, iniciada por volta dos anos 1990, se volta agora para a especificação e testagem na prática de resultados do paciente que sejam sensíveis à intervenção profissional. Uma vez que um diagnóstico de enfermagem é feito, especifica-se um resultado a ser alcançado e cria-se com isso uma dupla obrigação, a de intervir e, em seguida, avaliar a eficácia da intervenção realizada.

\section{Da teoria à prática assistencial e de pesquisa}

A ligação entre os elementos da prática profissional e a investigação científica pode ser identificada desde as primeiras expressões da pesquisa na área. Segundo a literatura, nas décadas de 1920 e 1930 começaram a ser publicados estudos de caso envolvendo a análise e a avaliação sistemáticas de um cliente ou grupo de clientes similares, para promover a compreensão acerca da situação e das intervenções de enfermagem que se faziam necessárias 9 .

Com o emprego dos estudos de caso, observaram-se o início do uso de instrumentos de coleta de informações sobre os pacientes, a análise e formulação de julgamentos clínicos sobre as informações coletadas e a diferenciação entre o que seria intervenção médica e de enfermagem, listadas separadamente ${ }^{7}$. Dessa forma, há uma indiscutível relação entre os estudos de caso e o aparecimento dos primeiros planos de cuidado de enfermagem. Assim como se pode afirmar que os estudos de caso foram os precursores dos planos de cuidado ${ }^{10}$, também se pode dizer que os planos de cuidado foram as primeiras expressões do que mais tarde se convencionou denominar Processo de Enfermagem.

0 Processo de Enfermagem é aplicável em uma ampla variedade de ambientes (instituições prestadoras de serviços de internação hospitalar ou de serviços ambulatoriais de saúde, escolas, associações comunitárias, fábricas, domicílios, entre outros); e em uma ampla variedade de situações clínicas em que as observações sobre as necessidades humanas da clientela acompanham, pari passu, o processo de tomada de decisão acerca do cuidado requerido e a avaliação posterior dos resultados que se obtêm com a ação/intervenção profissional ${ }^{1,3}$.

Exemplificaremos, a seguir, trabalhos desenvolvidos nos últimos dez anos no Programa de Pós-Graduação em Enfermagem - Centro de Ciências da Saúde da Universidade Federal da Paraíba (PPGENF-CCS/UFPB), em que se vinculam os elementos da prática profissional, inerentes ao Processo de Enfermagem (diagnósticos, intervenções e resultados de enfermagem), à pesquisa científica.

Antecedendo o diagnóstico de enfermagem, há exemplos de estudos metodológicos em que se desenvolveu escala psicométrica para avaliação do conceito de Imagem corpora/ ${ }^{11}$ ou instrumentos de coleta de dados a serem aplicados a clientes adultos em Clínica Médica ${ }^{12}$, a crianças de 0 a 5 anos em Clínica Pediátrica $^{13}$, a idosos atendidos no Programa de Saúde da Família $^{14}$, a clientes adultos em Clínica Cirúrgica ${ }^{15}$ e a recémnascidos internados em Unidade de Terapia Intensiva Neonatal ${ }^{16}$.

Os estudos que objetivavam desenvolver instrumentos de coleta de dados utilizaram como referencial a Teoria das Necessidades Humanas Básicas elaborada por Wanda de Aguiar Hor ta e percorreram etapas metodológicas similares: revisão de literatura sobre as necessidades humanas básicas e sua aplicação à clientela alvo do estudo; identificação, na literatura da área da saúde, de modo geral, e da Enfermagem, em 
particular, de indicadores empíricos das necessidades humanas básicas; avaliação, por grupo de enfermeira(0)s perita(o)s na área clínica específica, do grau de relevância dos indicadores empíricos identificados na literatura, sendo validados aqueles com índice de concordância $\geq 0,80$; construção da primeira versão do instrumento de coleta de dados; avaliação, pelo grupo de enfermeira(o)s perita(o)s, da estrutura, forma de apresentação e operacionalidade clínica do instrumento.

Com relação ao diagnóstico de enfermagem, os trabalhos desenvolvidos no PPGENF-CCS/UFPB percorreram duas vertentes metodológicas principais: 1) a validação clínica ou de conteúdo de características definidoras, fatores causais ou fatores de risco de diagnósticos específicos, como Padrão respiratório ineficaz ${ }^{17}$ e Risco para úlcera de pressão ${ }^{18}$; e 2) a construção, com base em modelos teóricos, sistemas conceituais ou teorias de enfermagem, de perfil diagnóstico para clientelas espećíicas, a exemplo de pacientes vítimas de trauma admitidos em CTI ${ }^{19}$, pacientes acometidos por infarto do miocárdio ${ }^{20}$, pacientes prostatectomizados ${ }^{21}$ ou pacientes internados em Clínica Médica e Clínica Cirúrgica22.

Ressalte-se que a elaboração de perfil diagnóstico de enfermagem para clientelas específicas aproxima-se do que 0 Conselho Internacional de Enfermagem-CIE denomina catálogos CIPE $^{\circledR}$ (Classificação Internacional para a Prática de Enfermagem), definidos como conjuntos de enunciados preestabelecidos de diagnósticos, intervenç̃es e resultados de enfermagem, a que se tem acesso rápido, de modo a facilitar a documentação da assistência prestada à clientela ${ }^{4}$.

Quanto às intervenções executadas pelos profissionais de enfermagem, pode-se, com o auxílio da pesquisa científica, não somente procurar conhecer seu custo operacional, como responder algumas questões que se impõem: que intervenções funcionam melhor para o alcance de um determinado resultado, diante de um diagnóstico de enfermagem específico; que intervenções são usualmente executadas em conjunto e que intervenções são usualmente executadas em determinadas áreas ou especialidades. As respostas a essas questões facilitariam o processo de determinação dos custos das intervenções de enfermagem, pois limitam o número àquelas mais usuais ao se cuidar de determinados grupos de clientes ou em determinados ambientes de cuidado. Identificadas, podese calcular um valor para elas, tendo por base o nível de complexidade, profissional envolvido (enfermeira, técnico ou auxiliar de enfermagem), tempo, quantidade e recursos/ equipamentos requeridos em sua execução ${ }^{23}$.

Nos últimos dez anos há, no PPGENF-CCS/UFPB, dois estudos que empregaram, especificamente, uma ligação entre os elementos diagnóstico e intervenção de enfermagem no processo de pesquisa. No primeiro deles ${ }^{24}$, após confirmar, à luz dos conceitos da Teoria das Necessidades Humanas Básicas de Horta, a presença do diagnóstico de enfermagem Mobilidade física prejudicada em trinta clientes hospitalizados, com foco nas características definidoras, apresenta-se uma proposta de intervenções de enfermagem, embasada na Classificação de Intervenções de Enfermagem (Nursing Interventions Classification-NIC) e na literatura da área. 0 segundo estudo ${ }^{25}$ aborda, sob o ponto de vista da Epidemiologia Social, diagnósticos de enfermagem estabelecidos para um grupo de pacientes portadores de AIDS e apresenta uma proposta de intervenções elaborada com base na CIPE ${ }^{\circledR}$.

Mais recentemente, buscando dar continuidade a atividades desenvolvidas junto à Associação Brasileira de Enfermagem (ABEn Nacional) e ao CIE, como responsáveis pela produção de um inventário vocabular de enfermagem em saúde coletiva ${ }^{26}$, desenvolveu-se uma pesquisa cuja finalidade era a de identificar os termos utilizados pelos diferentes componentes da equipe de enfermagem para se referir aos fenômenos/diagnósticos/ problemas e às ações/intervenções/prescrições de enfermagem, de modo a, não somente concorrer para a construção da CIPE ${ }^{\circledR}$, mas, sobretudo, elaborar um instrumental tecnológico, sensível a nossa realidade, para inserção em sistemas de informação da prática profissional. Esse projeto, concluído em 2005, gerou a elaboração de um segundo, cujo objetivo geral foi o de construir um banco de dados de enfermagem a serem introduzidos em sistemas de informação. Isso está sendo feito através da identificação de termos da linguagem profissional relacionados a fenômenos e ações de enfermagem e do mapeamento cruzado com as classificações de termos constantes na CIPE ${ }^{\circledR} \mathrm{e} \mathrm{em}$ outras terminologias da área.

Ressalte-se a importância da construção de um banco de dados que inclua termos relacionados a diagnósticos/ resultados e a aç̃̃es de enfermagem de modo a favorecer a utilização de uma linguagem comum e, ao mesmo tempo, integrar o conhecimento científico e o conhecimento prático da profissão. Considera-se que um banco assim construído pode contribuir sobremaneira para o registro sistemático dos elementos da prática - diagnósticos, intervenções e resultados de enfermagem; consequentemente, para 0 aumento de visibilidade e de reconhecimento profissional e, por outro lado, para uma possibilidade concreta de avaliação da prática de enfermagem.

\section{CONSIDERAÇÕES FINAIS}

Conforme se procurou argumentar neste trabalho, o Processo de Enfermagem tem representado o principal modelo metodológico para o desempenho sistemático da prática profissional, ou um instrumento tecnológico de que se lança mão para favorecer o cuidado, para organizar as condições necessárias à realização do cuidado e para documentar a prática profissional. Assim, ele deve ser compreendido como um meio, e não um fim em si mesmo.

Descreveu-se como a conceituação e a aplicação do Processo de Enfermagem evoluiu para o reconhecimento de que, além daquele julgamento estritamente vinculado à doença e suas complicaç̃̃es, os agentes da Enfermagem fazem outros tipos de julgamentos e agem com base nesses julgamentos. A 
aplicação deliberada e sistemática do Processo de Enfermagem pode acrescentar qualidade ao cuidado, melhorar a visibilidade e o reconhecimento profissional e representar uma possibilidade concreta de avaliação da prática profissional.

Seja considerado um modelo metodológico ou um instrumento tecnológico, o Processo de Enfermagem é extremamente complexo, assim como é complexa a noção de cuidado profissional de enfermagem. Implícitos e mesclados em seu desenvolvimento estão o conhecimento pessoal dos agentes da Enfermagem sobre as necessidades dos seres humanos quando se defrontam com alguma alteração em seu estado de saúde; o raciocínio lógico; o uso de novas e avançadas tecnologias, introduzidas a cada dia na área da saúde; a empatia; a experiência, habilidade e autenticidade no relacionamento interpessoal; a perícia ou destreza manual no desempenho das ações de cuidado; o comportamento ético, a sensibilidade e a expressão de emoções tais como compaixão e solidariedade humanas.

Assim, além da complexidade inerente ao próprio Processo de Enfermagem, podem ser identificadas outras dificuldades para sua implementação sistemática e efetiva na prática profissional, algumas relativas à formação profissional dos componentes da equipe de enfermagem eà organização de seu processo de trabalho; outras, às expectativas das instituiç̃es ou ambiente em que o cuidado profissional de enfermagem é realizado; outras, ainda, ao modo como a sociedade ou os gestores da saúde entendem a Enfermagem e o papel de seus exercentes.

Estudo de natureza histórica, abarcando o período de 1960 a 1986, aponta, com base em análise de artigos publicados em um periódico da área, causas das dificuldades para implementação do Processo de Enfermagem: a exigência do mercado de trabalho de uma maior complexidade de conhecimentos e especificidades do cuidado, de modo a acompanhar o avanço tecnológico na área médica; o desinteresse das instituições empregadoras no cuidado direto e no planejamento da assistência realizados pela(o) enfermeira(0), privilegiando-se $\mathrm{o}$ bom andamento (administrativo/gerencial) do serviço de enfermagem; o déficit de profissionais, acarretando número expressivo de pacientes a cuidar; a falta de destreza na execução de algumas técnicas, pondo em risco a avaliação da competência profissional; a dificuldade do cumprimento das ações prescritas pelo enfermeiro, devido ao despreparo dos atendentes de enfermagem, representantes majoritários da força de trabalho no período. Dessa forma, conclui-se que as dificuldades e resistências experimentadas não podem ser contabilizadas apenas à vontade dos profissionais, uma vez que estão permeadas por interesses os mais diversos, quase sempre antagônicos ${ }^{27}$.

Entretanto, longe de significar desestímulo, as dificuldades para a implantação sistemática e efetiva do Processo de Enfermagem, que se reconhece haver, indicam desafios a estimular nossa capacidade de reflexão e de ação. Precisamos rever os significados que, ao longo dos últimos anos ou décadas, estivemos atribuindo ao Processo de Enfermagem na prática profissional, seja no ensino, na assistência, na gerência/ administração ou na pesquisa de enfermagem. Se necessário, precisamos compreender/criar novos significados para ele, assumindo, em primeiro lugar, a premissa básica de que nós, os agentes da Enfermagem, somos seres humanos cuidando de seres humanos ou, como disse a Dra. Wanda de Aguiar Horta, gente que cuida de gente. A miríade de significados que a ação cuidadora pode assumir no contexto do Processo de Enfermagem é infindável. Essa é a ciência-arte-ideal que devemos cultivar.

\section{REFERÊNCIAS}

1. Garcia TR, Nóbrega MML. Processo de enfermagem e os sistemas de classificação dos elementos da prática profissional: instrumentos metodológicos e tecnológicos do cuidar. In: Santos I, Figueiredo NMA, Padilha MICS, organizadores. Enfermagem assistencial no

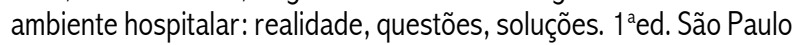
(SP): Atheneu; 2004. v. 2, p. 37-63.

2. Garcia JCR. Novas relações na transferência do conhecimento: patente, tecnologia, inovação. [tese de doutorado]. Rio de Janeiro (RJ): Programa de Pós-Graduação em Ciência da Informação/IBICT; 2004.

3. Garcia TR, Nóbrega MML, Carvalho EC. Processo de enfermagem: aplicação à prática profissional. Online Brazilian Journal of Nursing [on- line] 2004 ago; 3(2): [aprox.8 telas] Disponível em: http:// www.uff.br/nepae/objn302garciaetal.htm

4. International Council of Nurses (ICN). International Classification for Nursing Practice - ICNP ${ }^{\circledR}$ Version 1. Geneva; 2005.

5. Espirito Santo FH, Porto IS. De Florence Nightingale às perspectivas atuais sobre o cuidado de enfermagem: a evolução de um saber/ fazer. Esc Anna Nery 2006 dez; 10(3): p.539-46.

6. Pesut DJ, Herman JA. Clinical reasoning: the art and science of critical and creative thinking. Albany (NY): Delmar; 1999.

7. McGuire AD. The genesis and nature of nursing diagnosis. In: Carlson JH, Craft CA, McGuire AD, Popkess-Vawter S. Nursing diagnosis: a case study approach. Philadelphia (USA)): W. B. Saunders; 1991. p. 3-19.

8. Yura H, Walsh MB. The nursing process: assessment, planning, implementation and evaluation. New York (USA): Appleton-CenturyCrofts; 1967.

9. Burns N, Grove SK. The practice of nursing research: conduct, critique and utilization. $2^{\text {nd }}$ ed. Philadelphia (USA): W.B. Saunders; 1993.

10. Henderson VA. On nursing care plans and their history. Nurs Outlook 1973 June; 21 (6): 378-79.

11. Souto CMRM, Garcia TR. Construction and validation of a body image rating scale: a preliminary study. Int J Nurs Terminol Classif 2002; 13 (4): 117-26.

12. Virgínio NA, Nóbrega MML. Validação de instrumentos de coleta de dados de enfermagem para clientes adultos hospitalizados. Rev Bras Enferm 2004; 57(1): 53-56.

13. Silva KL, Nóbrega MML. Construção e validação de um instrumento de coleta de dados para crianças de 0-5 anos. Online Brazilian Journal 
of Nursing 2006 dez; 5(3): [aprox.8 telas] Disponível em: http:// www.uff.br/nepae/objn503silvanobrega.htm

14. Por to MLL, Nóbrega MML. Instrumento de coleta de dados para 0 atendimento do idoso no Programa de Saúde da Família. Revista de Enfermagem UFPE Online 2008 Jan; 2(1):1-8. Disponível em: http:// www.ufpe.br/revistaenfermagem/index.php/enfermagem/article/view/ $81 / 112$

15. Souza APMA. Construção e validação de um instrumento de coleta de dados para clientes adultos em Unidade Cirúrgica [dissertação de mestrado]. João Pessoa (PB): Centro de Ciências da Saúde / UFPB; 2007.

16. Gouvea JA. Construção e validação de um instrumento de coleta de dados para recém-nascidos internados na Unidade de Terapia Intensiva Neonatal do Hospital Universitário Lauro Wanderley [disser tação de mestrado]. João Pessoa (PB): Centro de Ciências da Saúde / UFPB; 2007.

17. Silva MFOC, Coler MS, Nóbrega MML. A culturally and developmentally-specific validation study of the NANDA nursing diagnosis: Ineffective breathing patterns. Proceedings of the $12^{\circ}$ Conference of the North American Nursing Diagnosis AssotiationNANDA; 1996; Pittsburg (PA), USA. Glendale (CA): CINAHL Information Systens; 1997. p. 252-56.

18. Silva MSML, Garcia TR. Fatores de risco para úlcera de pressão em pacientes acamados. Rev Bras Enferm 1998; 51 (4): 615-28.

19. Arruda AICG, Garcia TR. Diagnósticos de enfermagem relacionados à oxigenação, atribuídos a vítimas de trauma admitidos em CTI. Rev Bras Enferm 2000; 53 (3): 363-74.
20. Martins DL, Garcia TR. Perfil diagnóstico de enfermagem de pacientes acometidos por infarto do miocárdio. Online Brazilian Journal of Nursing [periódico on line] 2004 Aug; 3(2): [aprox.5 telas] Disponível em: http://www.uff.br/nepae/objn302martinsegarcia.htm.

21. Santana MS, Garcia TR. Diagnósticos de enfermagem em pacientes submetidos a prostatectomia. Nurs 2005; 90 (8): p. 528-33.

22. Silva ER, Fontes WD, Nóbrega MML. Diagnósticos de enfermagem de pacientes em unidade de internação em Clínica Médica e Clínica Cirúrgica. Temas em Saúde 2007; 7(1):11-167.

23. McCloskey JC, Bulechek GM. Nursing Interventions Classification (NIC): overview and current status. In: Oud N, organizer. Proceedings of the special conference of the Association of Common European Nursing Diagnoses, Interventions and Outcomes. Bern: Verlag Hans Huber; 2002. p.31-44

24. Pereira MA. Mobilidade física prejudicada em clientes hospitalizados e uma proposta de intervenções de enfermagem [dissertação de mestrado]. João Pessoa (PB): Centro de Ciências da Saúde/UFPB; 1997.

25. França UM. Diagnósticos de enfermagem e uma proposta de intervenções para pacientes com AIDS [dissertação de mestrado]. João Pessoa (PB): Centro de Ciências da Saúde/UFPB; 1999.

26. Garcia TR, Nóbrega MMML. Phenomena and actions of collective health nursing in Brazil. Nurs Lead Forum 2003; 7 (3): 116-20.

27. Kletemberg DF, Siqueira MD, Mantovani MF. Uma história do processo de enfermagem nas publicações da Revista Brasileira de Enfermagem no período 1960-1986. Esc Anna Nery Rev Enferm 2006 dez; 10(3): p.478-86. 